\title{
Training time and quality of smartphone-based anterior segment screening in rural India
}

\author{
This article was published in the following Dove Press journal: \\ Clinical Ophthalmology \\ 14 July 2017 \\ Number of times this article has been viewed
}

\author{
Cassie A Ludwig' \\ Megan R Newsom' \\ Alexandre Jais' \\ David J Myung',2 \\ Somasheila I Murthy ${ }^{3}$ \\ Robert T Chang' \\ 'Department of Ophthalmology, \\ The Byers Eye Institute, Stanford \\ University School of Medicine, \\ 2Department of Ophthalmology, VA \\ Palo Alto Health Care System, Palo \\ Alto, CA, USA; ${ }^{3}$ Cornea and Anterior \\ Segment Service, LV Prasad Eye \\ Institute, Kallam Anji Reddy Campus, \\ Hyderabad, India
}

Objective: We aimed at evaluating the ability of individuals without ophthalmologic training to quickly capture high-quality images of the cornea by using a smartphone and low-cost anterior segment imaging adapter (the "EyeGo" prototype).

Methods: Seven volunteers photographed 1,502 anterior segments from 751 high school students in Varni, India, by using an iPhone 5S with an attached EyeGo adapter. Primary outcome measures were median photograph quality of the cornea and anterior segment of the eye (validated Fundus Photography vs Ophthalmoscopy Trial Outcomes in the Emergency Department [FOTO-ED] study; 1-5 scale; 5, best) and the time required to take each photograph. Volunteers were surveyed on their familiarity with using a smartphone (1-5 scale; 5 , very comfortable) and comfort in assessing problems with the eye (1-5 scale; 5 , very comfortable). Binomial logistic regression was performed using image quality (low quality: $<4$; high quality: $\geq 4$ ) as the dependent variable and age, comfort using a smartphone, and comfort in assessing problems with the eye as independent variables.

Results: Six of the seven volunteers captured high-quality (median $\geq 4 / 5$ ) images with a median time of $\leq 25$ seconds per eye for all the eyes screened. Four of the seven volunteers demonstrated significant reductions in time to acquire photographs $(P 1=0.01, P 5=0.01, P 6=0.01$, and $P 7=0.01$ ), and three of the seven volunteers demonstrated significant improvements in the quality of photographs between the first 100 and last 100 eyes screened $(P 1<0.001, P 2<0.001$, and $P 6<0.01$ ). Self-reported comfort using a smartphone (odds ratio $[\mathrm{OR}]=1.25 ; 95 \% \mathrm{CI}=1.13$ to 1.39 ) and self-reported comfort diagnosing eye conditions ( $\mathrm{OR}=1.17 ; 95 \% \mathrm{CI}=1.07$ to 1.29 ) were significantly associated with an ability to take a high-quality image $(\geq 4 / 5)$. There was a nonsignificant association between younger age and ability to take a high-quality image.

Conclusion: Individuals without ophthalmic training were able to quickly capture a highquality magnified view of the anterior segment of the eye by using a smartphone with an attached imaging adapter.

Keywords: EyeGo, macrolens, screening, usability, smartphone, adapter, paxos, healthy scholars

\section{Introduction}

Anterior segment photography is an important part of a complete mobile eye examination as it provides direct information about the health of the eyelids, eyelashes, lacrimal system, conjunctiva, sclera, cornea, anterior chamber, iris, and lens. We used a low-cost, custom-made mobile phone attachment for anterior segment imaging (referred to herein as the "EyeGo" adapter) described in detail elsewhere" that was an early prototype of a commercially available ophthalmic imaging adapter for smartphones called Paxos Scope $^{\text {TM }}$ (DigiSight Technologies, San Francisco, CA, USA). The EyeGo consists of three parts: a light-emitting diode light source, a macrolens, and an apparatus that holds the smartphone - aligning the macrolens and the smartphone camera (Figure 1). ${ }^{1}$
Correspondence: Robert T Chang Department of Ophthalmology, The Byers Eye Institute, Stanford University School of Medicine, 2452 Watson Ct, MC 5353, Palo Alto, CA 94303, USA Email rchang3@stanford.edu 

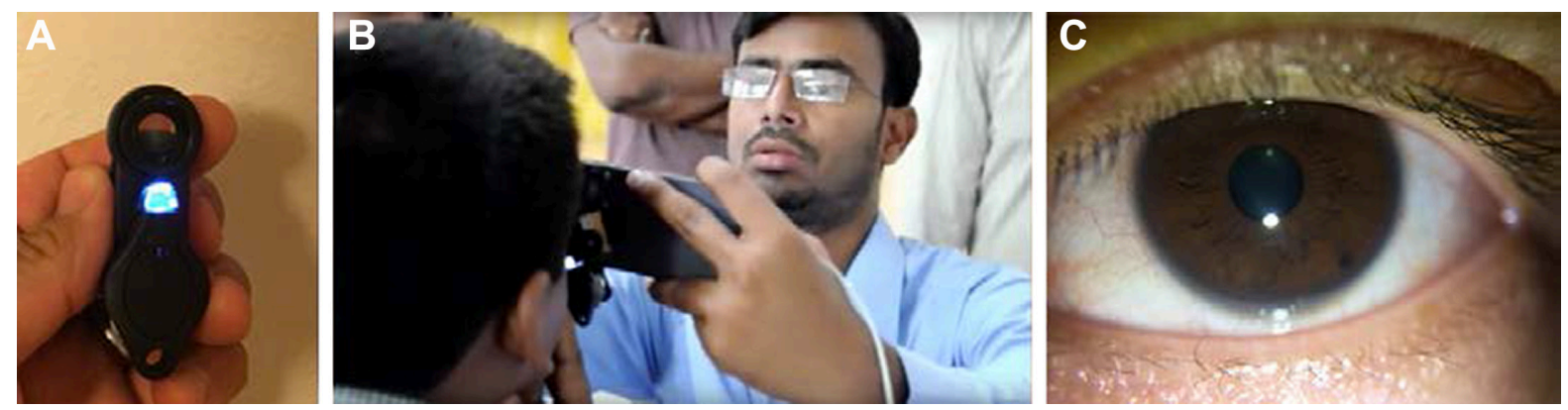

Figure I The EyeGo adapter.

Notes: (A) The EyeGo adapter for anterior segment imaging: a smartphone adapter consisting of an external LED light source and a macrolens. (B) A health fair screening volunteer capturing images of the anterior segment for a high school student by using the EyeGo adapter prototype. (C) A photograph taken by the EyeGo adapter of a normal eye.

Abbreviation: LED, light-emitting diode.

The EyeGo magnifies the cornea and surface aspects of the eye, enabling front and back of the eye imaging, and its simple design makes it an ideal screening tool for performing mobile eye examinations. ${ }^{1,2}$

Although Myung et al demonstrated that the EyeGo is easy for medical professionals to use, the device remains relatively untested by users without training in eye care. ${ }^{1-4}$ Consequently, our primary aim was to evaluate the ability of untrained users to quickly capture high-quality images of the front of the eye, and our secondary aim was to assess the relationship between a user's age, comfort using a smartphone, and comfort in assessing problems with the eye and the user's ability to capture high-quality images of the front of the eye.

\section{Methods}

Research was performed ethically and in accordance with the Institutional Review Board of Stanford University and L V Prasad Eye Institute, both of which approved this research. Verbal informed consent was obtained from the parent of each minor participating in Healthy Scholars Screening, and the study was conducted in a Health Insurance Portability and Accountability Act (HIPAA)-compliant manner. All research adhered to the tenets of the Declaration of Helsinki. All image acquisition and transmittal were handled with strict attention to the confidentiality of personal data in accordance with the Data Protection Act of 1998 and Access to Health Records of 1990.

The iPhone 5S (Apple Inc., Cupertino, CA, USA) used to capture images was encrypted using the Stanford University Mobile Device Management application. ${ }^{5}$ Images were uploaded from the phone to research electronic data capture (REDCap) tools hosted at Stanford University after the event. The study data were input to and managed using REDCap.
REDCap is a secure, web-based application designed to support data capture for research studies, providing 1) an intuitive interface for validated data entry; 2) audit trails for tracking, manipulation, and export procedures; 3 ) automated export procedures for seamless data downloads to common statistical packages; and 4) procedures for importing data from external sources.

The EyeGo screening study included all students presenting at the Center for Development (CCD) in Varni for the Healthy Scholars Screening over 4 consecutive days in August 2014, with a total of 24.4 hours of screening with the EyeGo. Students cycled through 5 health stations including thyroid, vision, hearing, dental, and girl's health screening. Following the vision station, students were screened with the EyeGo. In total, 111 students were screened sequentially on Day 1 by 2 different users (time spent specifically on EyeGo screening: 4 hours, 55 minutes), 224 by 3 users on Day 2 (7 hours, 47 minutes), 214 by 1 user on Day 3 ( 5 hours, 49 minutes), and 217 by 3 users on Day 4 ( 5 hours, 42 minutes; 1 user had also worked on Day 3). All students from two high schools were offered this free health screening. We excluded 15 students who were screened by a user who had difficulty using the device quickly (the individual had never used a smartphone) and who was requested to switch roles at the screening due to time constraints. Age was recorded from Healthy Scholars Screening forms. Both eyes of all 751 patients were imaged.

Patients underwent visual acuity testing and extraocular movement testing and then were imaged by one of seven Healthy Scholars volunteers with varying levels of training and education (eg, undergraduate student, undergraduate degree holder, land surveyor, lab technician, and multipurpose health worker). Healthy Scholars volunteers already present at the screening had been randomly recruited to 
perform EyeGo imaging, based on their availability during the 5 days of screening.

Images were taken indoors in a large gymnasium with fluorescent lighting. Users could take additional photographs of each eye if they felt that their first was unsuccessful for any of the following reasons: if the patient or his/her eye or eyelid moved unexpectedly or if the user lost hand positioning over the eye as he/she was taking the photograph. In general, 1-3 images were obtained for each eye of each patient, and the highest quality image (normal or with pathology) was used for the analysis, as determined by the grader post hoc.

Healthy Scholars volunteers were given brief $(<5$ minutes $)$ standard instructions on how to take photographs by using the EyeGo. ${ }^{1}$ Users were instructed to hold the iPhone $5 \mathrm{~S}$ with two hands: one hand held the phone sideways with the third digit and thumb, whereas the fourth and fifth digits stabilized the phone against the patient's forehead, and the other hand further stabilized the phone. The index finger of the hand closest to the button on the camera was used to take a photograph. Users were given real-time feedback on their first 10 images by an experienced user (CL). Care was taken in all cases to prevent contact between the phone and adapter and the patient's skin. In case contact was made, the adapter and phone were wiped clean with an alcohol swab, and the adapter itself was submerged in soap and water or another cleaning solution.

Prior to imaging, users were asked their highest level of training/education in addition to two survey questions: 1) How comfortable do you feel using a smartphone? and 2) how comfortable do you typically feel while assessing problems with the eye? Users responded on a 5-point Likert scale (Table 1).

One medical student grader de-identified and graded all images by using a 5-point scale previously validated for nonmydriatic imaging in the FOTO-ED study by Lamirel et al. ${ }^{6}$ Images were graded using the following criteria from the FOTO-ED study: 1) The quality is inadequate for any diagnostic purpose; 2) grader was unable to exclude all emergent findings; 3 ) grader was only able to exclude emergent findings; 4) the quality is not ideal, but grader was able to exclude subtle findings; and 5) image was of ideal quality. ${ }^{6}$ Emergent findings from anterior imaging include corneal abrasions/lacerations/ulcers, episcleritis, scleritis, chemical burns, foreign bodies, hyphema, hypopyon, and traumatic injury. An example of a subtle finding for anterior imaging includes fine detail of the iris. High-quality images were defined as images with a grade of $\geq 4 / 5$. This scale was appropriate for determining the utility of the EyeGo in ruling out emergent findings.
Table I Baseline characteristics of EyeGo users $(n=7)$ and their relationship with image quality

\begin{tabular}{|c|c|c|}
\hline Characteristic & n (\%) & $\begin{array}{l}\text { Odds ratio } \\
\text { ( } 95 \% \text { confidence } \\
\text { interval) }\end{array}$ \\
\hline $\mathrm{Age}^{\mathrm{b}}$ - median (interquartile range) & $20(20,36)$ & $0.915^{c}(0.77$ to 1.09$)$ \\
\hline \multicolumn{3}{|c|}{ Highest level of training/education } \\
\hline Undergraduate student & 2 of $7(28.6)$ & \\
\hline Undergraduate degree & I of $7(14.3)$ & \\
\hline Lab technician & I of $7(14.3)$ & \\
\hline $\begin{array}{l}\text { Multipurpose health worker } \\
\text { (female) }\end{array}$ & 2 of $7(28.6)$ & \\
\hline Land surveyor & I of $7(\mid 4.3)$ & \\
\hline \multicolumn{3}{|c|}{ "How comfortable do you feel using a smartphone?"d } \\
\hline Very comfortable & 4 of $7(57.1)$ & 1.25 (1.13 to 1.39$)$ \\
\hline Somewhat comfortable & I of $7(14.3)$ & \\
\hline Neutral & I of $7(14.3)$ & \\
\hline Somewhat uncomfortable & I of $7(14.3)$ & \\
\hline Very uncomfortable & 0 of $7(0.0)$ & \\
\hline \multicolumn{3}{|c|}{$\begin{array}{l}\text { "How comfortable do you typically feel while assessing problems with } \\
\text { the eye?"d }\end{array}$} \\
\hline Very comfortable & 2 of $7(28.6)$ & I.I7 (1.07 to I.29) \\
\hline Somewhat comfortable & I of $7(14.3)$ & \\
\hline Neutral & 3 of 7 (42.9) & \\
\hline Somewhat uncomfortable & I of $7(14.3)$ & \\
\hline Very uncomfortable & 0 of $7(0.0)$ & \\
\hline
\end{tabular}

Notes: astimated by using a binary regression model with image quality $<4$ or $\geq 4$ as the outcome variable and age, comfort using a smartphone, and comfort in assessing problems with the eye as predictors; ${ }^{b}$ median age was used, as the distribution was non-normal when tested by using the Shapiro-Wilk test statistic; 'calculated by 10 -year intervals in age; ${ }^{~}$ EyeGo users were asked these two questions prior to screening.

Images were reviewed on a 15.4-inch backlit display monitor with in-plane switching technology that facilitates viewing at a broad angle at which images can be viewed without deterioration of color and brightness (MacBook Pro, resolution $=2,880 \times 1,800$ at 220 pixels per inch). The reviewer could adjust monitor contrast and brightness for image review.

Data were analyzed by using statistical methods for prospective cohort designs. Median difference was used as the basic summary measure of change in quality and time by experience. Odds ratios (ORs) were used as the basic summary measure of association between age, comfort using a smartphone, and comfort in assessing problems with the eye and image quality. All the analyses were conducted by using Statistical Analysis Software Enterprise Guide Version 6.1 (Cary, NC, USA).

Variables were first graphically examined for normal distributions and assessed for outliers to determine the appropriate statistical test. The Shapiro-Wilk test was used to test for normal distribution. Measures of central tendency and variation as appropriate to the study variable and its 
distribution were used to describe the study population. Binomial logistic regression using image quality (ie, $<4$ or $\geq 4$ ) as the dependent variable was used to obtain ORs, with age, comfort using a smartphone, and comfort in assessing problems with the eye as predictors.

The Wilcoxon rank sum test was used to compare the time each user took to take each of the first 100 photographs and the second 100 photographs as well as the quality of the first 100 and second 100 photographs. This test was chosen as time and quality were not normally distributed. This allowed us to determine the unadjusted $P$-value for the difference between the groups. Statistical tests had a twotailed $\alpha$ of 0.05 .

\section{Results}

Eight volunteers from the Healthy Scholars organization imaged the eyes of a total of 766 subjects. One user did not complete imaging of 100 subjects due to difficulty using the EyeGo quickly and time constraints from other segments of the health screening; therefore, the user and all 15 subjects imaged by this user were excluded from the study. Table 2 reports baseline characteristics of the subjects imaged. The median age of participants was 12.5 years. Imaging and external examination assisted in recording oculodermal melanocytosis, arcus, and conjunctival injection (Figure 2A-D) in addition to stye, ptosis, and blepharitis. Only $2.3 \%$ of subjects reported ocular complaints prior to

Table 2 Baseline characteristics of subjects screened by Healthy Scholars

\begin{tabular}{|c|c|}
\hline Baseline characteristics & n (\%) \\
\hline Age $^{\mathrm{a}}$ - median (interquartile range) & $12.5(\mid 1.5,13.7)$ \\
\hline \multicolumn{2}{|l|}{ Presenting ocular complaints } \\
\hline Watering & 9 of 751 (I.2) \\
\hline Blurred vision & 6 of $751(0.8)$ \\
\hline Ocular pain & 5 of $75 I(0.7)$ \\
\hline Photophobia & 2 of $75 \mid(0.3)$ \\
\hline Floaters & 2 of $751(0.3)$ \\
\hline Total & 17 of $75 \mid(2.3)$ \\
\hline \multicolumn{2}{|c|}{ Anterior segment findings on examination } \\
\hline Decreased visual acuity & 90 of 751 (12.0) \\
\hline Stye & I of $75 \mid(0.1)$ \\
\hline Strabismus & I of $75 \mid(0.1)$ \\
\hline Ptosis & I of $75 \mid(0.1)$ \\
\hline Blepharitis & I of 75 I $(0.1)$ \\
\hline Periorbital wound & I of 75 I $(0.1)$ \\
\hline Oculodermal melanocytosis & I of $75 \mid(0.1)$ \\
\hline Arcus & I of $75 \mid(0.1)$ \\
\hline Conjunctival injection & $\mid$ of $75 \mid(0.1)$ \\
\hline Total & 98 of $751(13.0)$ \\
\hline
\end{tabular}

Notes: aedian age was used, as the distribution was non-normal when tested by using the Shapiro-Wilk test statistic; $n=751$. examination, with watering $(1.2 \%)$ being the most common complaint. Ophthalmic examination revealed ocular findings in $13.0 \%$ (98 of 751$)$ of subjects with decreased visual acuity being the most common (90 of $751 ; 12.0 \%$ ).

Table 1 lists baseline characteristics of the seven anterior adapter EyeGo users. The median age of users was 20 years, with a range of 19-38 years. There was a nonsignificant association between younger age and higher quality of images with a reduction in the odds of high-quality images for each additional 10 years of age $(\mathrm{OR}=0.92 ; 95 \% \mathrm{CI}=0.77$ to 1.09$)$. All seven of these individuals answered two questions regarding their comfort in administering tests prior to screening. In response to the first question "How comfortable do you feel using a smartphone?" the majority felt "very comfortable" $(57.1 \%)$. In response to the second question "How comfortable do you typically feel while assessing problems with the eye?" most reported "neutral" (42.9\%). For every 1 -point increase in comfort using a smartphone on a 5-point Likert scale, users experienced a $25 \%$ increase (OR $=1.25$; $95 \% \mathrm{CI}=1.13$ to 1.39 ) in the odds of taking a high-quality image $(\geq 4 / 5)$. For every 1 -point increase in comfort in assessing problems with the eye on a 5-point Likert scale, users experienced a $17 \%$ increase $(\mathrm{OR}=1.17 ; 95 \% \mathrm{CI}=1.07$ to 1.29 ) in the odds of taking a high-quality image.

Three users of the EyeGo adapter for anterior segment imaging achieved a median image quality of 5 (the highest value) for the first 100 eyes imaged and continued to have a median image quality of 5 for the second 100 eyes imaged with no significant change $(P 3=0.20, P 4=0.67$, and $P 5=0.70$; Table 3). Three users achieved an improvement in image quality between the first 100 eyes imaged and second 100 eyes imaged $(P 1<0.001, P 2<0.001$, and $P 6<0.001)$. A total of six users $(85.7 \%)$ achieved a median image quality of 5 for the last 100 eyes imaged.

Four users experienced a significant decrease $(P 1=0.01$, $P 5=0.01, P 6=0.01$, and $P 7=0.01)$ in median time between the first and second 100 eyes imaged (Table 3). Another user experienced a nonsignificant decrease in median time $(P 3=0.13)$. Two users experienced an increase in median time between the first and second 100 eyes imaged - neither of these increases was significant $(P 2=0.15$ and $P 4=0.15)$.

\section{Discussion}

Our study demonstrates that volunteers with no prior ophthalmic training and minimal device training $(<5$ minutes and feedback with the first 10 patients) could capture high-quality images of the cornea and front of the eye for 1,502 eyes with a median time of $\leq 25$ seconds per eye. Six of seven 

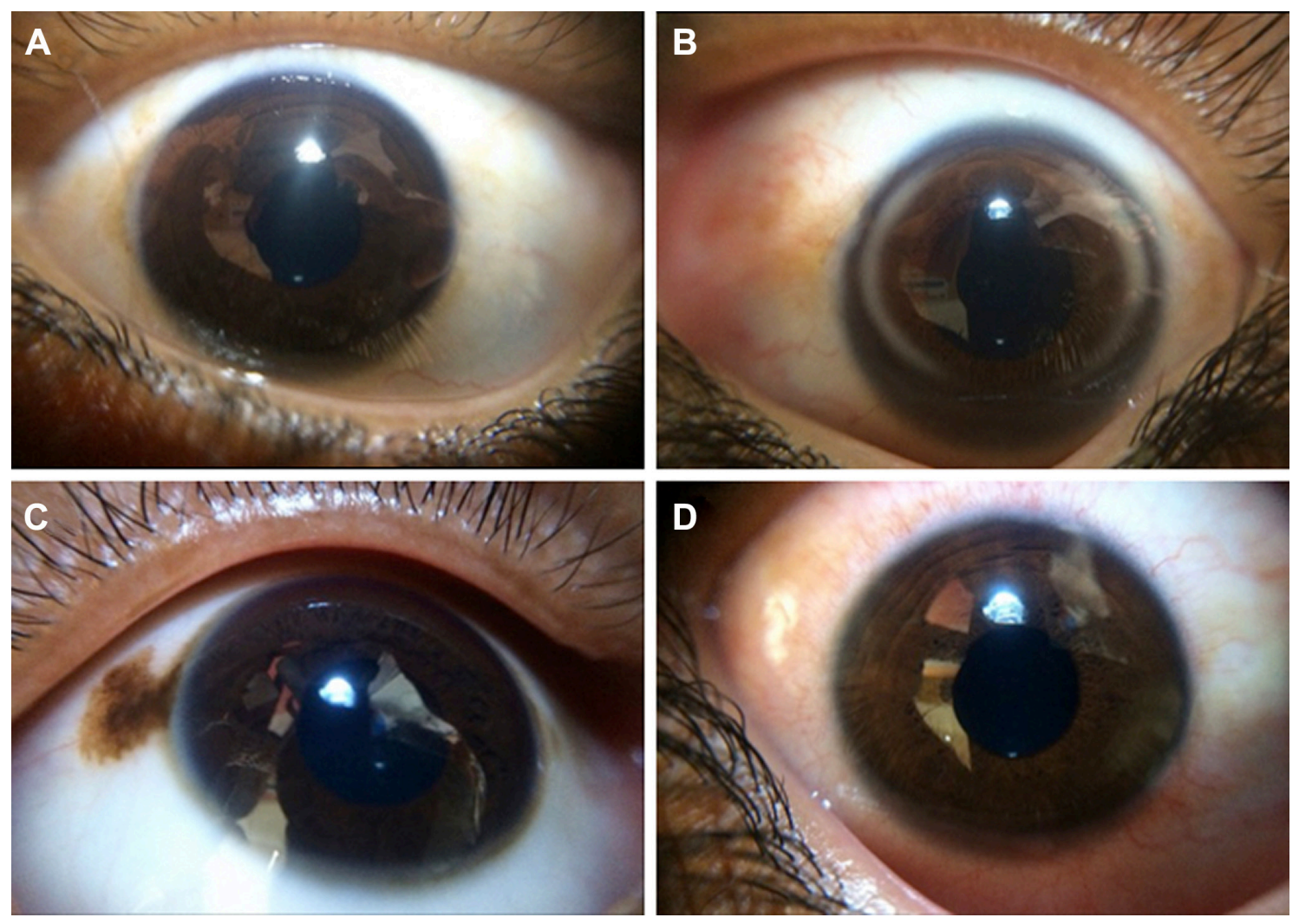

Figure 2 Photographs of the ocular surface taken by using the EyeGo adapter for anterior segment imaging. Reflections present due to suboptimal lighting conditions. Notes: (A) Normal. (B) Arcus resulting from the deposition of lipids in the peripheral cornea. (C) Oculodermal melanocytosis associated with glaucoma. (D) Conjunctival injection.

volunteers captured high-quality (median $\geq 4 / 5$ ) images of the eye at baseline. EyeGo users with a baseline image quality $<5$ underwent an improvement in median image quality with experience. Furthermore, most users experienced a significant reduction in median time to capture images. In addition, we found that a user's self-reported comfort using a smartphone and in assessing eye problems increased the odds of the user capturing high-quality images. Lastly, 13.0\% (98 of 751) of students had ocular findings on examination with decreased visual acuity being the most common (90 of $751 ; 12.0 \%)$ followed by 1 each $(0.1 \%)$ of stye, strabismus, ptosis, blepharitis, periorbital wound, oculodermal melanocytosis, arcus, and conjunctival injection.

The prevalence of anterior segment ocular morbidity detected in our study (13.0\%) was similar to that found in a study that conducted ophthalmologic screening examinations of 1,157 students aged 6-16 years at a municipal (12.92\%) and private school (9.82\%) in Pune City, Maharashtra, India. ${ }^{7}$

Table 3 Changes in time taken per eye and change in the quality of photographs taken with experience

\begin{tabular}{|c|c|c|c|c|c|c|c|c|}
\hline \multirow[t]{2}{*}{ User } & \multicolumn{2}{|c|}{ First 100 eyes } & \multicolumn{2}{|c|}{ Last 100 eyes } & \multicolumn{2}{|c|}{ Difference } & \multicolumn{2}{|l|}{$P$-value ${ }^{b}$} \\
\hline & $\begin{array}{l}\text { Median } \\
\operatorname{time}^{\mathrm{a}}(\mathrm{s})\end{array}$ & $\begin{array}{l}\text { Median } \\
\text { quality }\end{array}$ & $\begin{array}{l}\text { Median } \\
\text { time (s) }\end{array}$ & $\begin{array}{l}\text { Median } \\
\text { quality }\end{array}$ & Time (s) & Quality & Time (s) & Quality \\
\hline I & 25.0 & 3.0 & 17.5 & 4.0 & -7.5 & 1.0 & 0.01 & $<0.001$ \\
\hline 2 & 18.0 & 4.0 & 20.0 & 5.0 & 2.0 & 1.0 & 0.15 & $<0.001$ \\
\hline 3 & 20.0 & 5.0 & 18.0 & 5.0 & -2.0 & 0.0 & 0.13 & 0.20 \\
\hline 4 & 22.0 & 5.0 & 24.0 & 5.0 & 2.0 & 0.0 & 0.15 & 0.67 \\
\hline 5 & 12.0 & 5.0 & 10.0 & 5.0 & -2.0 & 0.0 & 0.01 & 0.70 \\
\hline 6 & 25.0 & 4.0 & 15.5 & 5.0 & -9.5 & 1.0 & $<0.001$ & $<0.01$ \\
\hline 7 & 22.0 & 4.0 & 16.0 & 5.0 & -6.0 & 1.0 & 0.01 & 0.11 \\
\hline
\end{tabular}

Notes: Median time and quality were used, as distributions were non-normal when tested by using the Shapiro-Wilk test statistic; bStatistical significance was measured by using the Wilcoxon rank sum test. 
However, it was lower than the overall prevalence of ocular morbidity found through questionnaires provided to $1,561 \mathrm{stu}-$ dents aged 6-16 years in Shimla, Himachal, India (31.6\%). ${ }^{8}$

Anterior segment imaging is a necessary part of a complete mobile eye examination as it can detect disease of the eyelids, eyelashes, lacrimal system, conjunctiva, sclera, cornea, anterior chamber, iris, and lens. ${ }^{1}$ Currently, several components of a comprehensive eye examination can be completed by using a smartphone and smartphone applications or hardware attachments: visual acuity, ${ }^{9}$ Amsler grid testing, ${ }^{10}$ contrast testing, ${ }^{10}$ color vision assessment, ${ }^{10}$ and refraction. ${ }^{11,12}$ Applications for the assessment of pupillary response, extraocular movements, and visual field testing are not yet available but will likely become available in the near future. With a rise in smartphone owners in developing nations from a median of $21 \%$ in 2013 to $37 \%$ in 2015 , the movement toward a smartphone-centered ocular examination increases accessibility in terms of both cost and convenience to underserved populations. ${ }^{13}$ This is in contrast to established methods for nonmobile anterior segment imaging that require slit lamps and/or advanced training with more expensive devices. ${ }^{14-16}$ Detection of ocular disease is critical as the World Health Organization estimates that $80 \%$ of worldwide ocular pathology is curable and preventable. ${ }^{17}$ Access to low-cost and easy-to-use tools for performing ocular screening allows for such detection. We confirm here that the EyeGo presents an easy-to-use alternative to these methods. Further, images taken with the EyeGo allow for remote reading of images through a secure, encrypted email or HIPAA-compliant smartphone applications.

Our study was limited by the exclusion of one user who could not finish the screening due to unfamiliarity with and difficulty using the smartphone. Comfort using smartphones was significantly associated with the ability to take high-quality images; therefore, being comfortable using a smartphone contributes to the ease of use of the EyeGo. In addition, the use of the Likert scale in our survey data has disadvantages as it limits respondents to 5 choices, often not truly equidistant in value. Lastly, our participant sample size - and therefore user sample size, as each user was required to image 100 students to have been included in the study - was limited by the number of students attending the Healthy Scholars Screening.

Our study demonstrates the feasibility of adding anterior segment imaging to a mobile eye examination with the EyeGo attachment. In the setting of this health screen, anterior segment imaging allowed for the detection and documentation of pathology that may otherwise have been missed during visual acuity screening. Anterior segment imaging fits seamlessly into a comprehensive eye screening otherwise including vision screening, refraction, pupil assessment, posterior photographs, visual field testing, and imaging with ocular coherence tomography. The low cost, ease of use, and portability of the EyeGo enable it to provide anterior and posterior segment imaging in any setting - from a doctor's office to a gymnasium in rural Varni.

\section{Acknowledgments}

This research would not have been possible without the help of the Healthy Scholars Foundation, Raman and Srini Madala, Lily Truong, the Madala Charitable Trust, and CCD Varni in organizing the health screen and supporting all aspects of the study. Thank you to the faculty and staff at L V Prasad Eye Institute for their general support and enthusiasm for this project. Thank you also to the staff at Nizamabad Lion's Eye Club for volunteering their time to complete the remainder of the vision screening examination. We greatly appreciate the time of the Healthy Scholars volunteers involved in imaging students: Srujan Mara, Bojja Srija, Sangeetha Sriramwar, Boyapati Ramkishore, Alpana Chintam, Sridhar Bodapati, Mohammad Hussain, and Venkatalaxmi. Cassie A Ludwig was funded by the TL1 Clinical Research Training Program (NIH TL1 TR 001084) in addition to the Stanford University Medical Scholars Research Foundation. David J Myung and Robert T Chang received support from the Stanford Society of Physician Scholars Grant in addition to the Stanford SPECTRUM/Biodesign Research Grant.

\section{Disclosure}

Robert T Chang, Alexandre Jais, and David J Myung are patent holders of the smartphone ophthalmic imaging system discussed. David J Myung is a consultant to DigiSight Technologies. Cassie A Ludwig, Megan Newsom, and Somasheila I Murthy report no conflicts of interest in this work.

\section{References}

1. Myung D, Jais A, He L, Chang R. Simple, low-cost smartphone adapter for rapid, high quality ocular anterior segment imaging: a photo diary. J Mob Technol Med. 2014;3(1):2-8.

2. Myung D, Jais A, He L, Blumenkranz M, Chang R. 3D printed smartphone indirect lens adapter for rapid, high quality retinal imaging. JMob Technol Med. 2014;3(1):9-15.

3. Ludwig CA, Murthy SI, Pappuru RR, Jais A, Myung DJ, Chang RT. A novel smartphone ophthalmic imaging adapter: user feasibility studies in Hyderabad, India. Indian J Ophthalmol. 2016;64(3):191-200.

4. Toy BC, Myung DJ, He L, et al. Smartphone-based dilated fundus photography and near visual acuity testing as inexpensive screening tools to detect referral warranted diabetic eye disease. Retina. 2016;36(5): $1000-1008$. 
5. Harris PA, Taylor R, Thielke R, Payne J, Gonzalez N, Conde J. Research electronic data capture (REDCap) - a metadata-driven methodology and workflow process for providing translational research informatics support. J Biomed Inform. 2009;42(2):377-381.

6. Lamirel C, Bruce BB, Wright DW, Delaney KP, Newman NJ, Biousse V. Quality of nonmydriatic digital fundus photography obtained by nurse practitioners in the emergency department: the FOTO-ED study. Ophthalmology. 2012;119(3):617-624.

7. Kumar P, Pore P, Dixit A, Jha A, Ahmad A, Chauhan N. Demographic profile of ocular morbidity in school children in India. Sch J App Med Sci. 2013;1(5):645-652.

8. Gupta M, Gupta BP, Chauhan A, Bhardwaj A. Ocular morbidity prevalence among school children in Shimla, Himachal, North India. Indian J Ophthalmol. 2009;57(2):133-138.

9. Peek Vision [homepage on the Internet]. London: Peek Vision Ltd.; c2016-2017 [updated 2017 April 13; cited 2017 February 23]. Available from: http://www.peekvision.org/. Accessed June 21, 2017.

10. Eye Handbook [homepage on the Internet] Kansas City: Eye Handbook; c2009-2017 [updated 2017 June 6; cited 2017 February 23]. Available from: http://www.eyehandbook.com/. Accessed June 21, 2017.

11. Opternative [homepage on the Internet] Chicago: Opternative, Inc.; c2013-2017; [updated 2017 June 21; cited 2017 February 23]. Available from: https://www.opternative.com/. Accessed June 21, 2017.
12. EyeNetra [homepage on the Internet] Somerville: EyeNetra, Inc.; c2014-2017; [updated 2017 June 14; cited 2017 February 23]. Available from: https://eyenetra.com/. Accessed June 21, 2017.

13. Poushter J. Smartphone Ownership and Internet Usage Continues to Climb in Emerging Economies: But advanced economies still have higher rates of technology use. Pew Research Center [serial on the Internet]. 2016 February. [cited 2017 February 23]. Available from: http://www.pewglobal.org/2016/02/22/smartphone-ownership-andinternet-usage-continues-to-climb-in-emerging-economies/. Accessed June 21, 2017.

14. Chakrabarti D. Application of mobile technology in ophthalmology to meet the demands of low-resource settings. J Mob Technol Med. 2012; 1(4S):1-3.

15. Lord R, Shah VA, San Filippo AN, Krishna R. Novel uses of smartphones in ophthalmology. Ophthalmology. 2010;117(6):1274.e1273.

16. Zvornicanin E, Zvornicanin J, Hadziefendic B. The use of smart phones in ophthalmology. Acta Inform Med. 2014;22(3):206-209.

17. Visual impairment and blindness: Fact Sheet $\mathrm{N}^{\circ} 282$ [serial on the Internet]. Geneva: World Health Organization; 2014. Available from http://www.who.int/mediacentre/factsheets/fs282/en/. Accessed February 13, 2017.
Clinical Ophthalmology

\section{Publish your work in this journal}

Clinical Ophthalmology is an international, peer-reviewed journal covering all subspecialties within ophthalmology. Key topics include: Optometry; Visual science; Pharmacology and drug therapy in eye diseases; Basic Sciences; Primary and Secondary eye care; Patient Safety and Quality of Care Improvements. This journal is indexed on

Submit your manuscript here: http://www.dovepress.com/clinical-ophthalmology-journal

\section{Dovepress}

PubMed Central and CAS, and is the official journal of The Society of Clinical Ophthalmology (SCO). The manuscript management system is completely online and includes a very quick and fair peer-review system, which is all easy to use. Visit http://www.dovepress.com/ testimonials.php to read real quotes from published authors. 